MS45-O5 Deriving a chemical context for protein-bound monosaccharides

Jon Agirre ${ }^{1}$, Kevin Cowtan ${ }^{1}$

1. York Structural Biology Laboratory, Department of Chemistry, The University of York, England

email: jon.agirre@york.ac.uk

Any attempt at gaining chemical information from a public structural biology repository such as the Protein Data Bank (PDB) should deal with the fact that many deposited structures contain errors. Remediation efforts to date have, with the exception of PDB-REDO [1], focused on the correction of naming problems, while the atomic coordinates remain untouched until a new entry supersedes them. Therefore, scientists are encouraged to use as many validation tools as possible in order to arrive at meaningful conclusions.

Structural fingerprinting is a process that, starting from a set of deposited atomic models, extracts a chemical context for a target non-protein compound. The resulting fingerprint can be used for detecting the compound's features and context in an electron density map, thus allowing for its interpretation in terms of atomic positions. The approach, which is already an integral part of the Nautilus software for the automated detection and modelling of nucleic acid [2], has recently been extended to work with cyclic carbohydrates. However, the prerequisite systematic analysis of the monosaccharide-containing entries in the PDB archive has uncovered new issues involving ring conformation, to be added to the already known nomenclature problems [3]. A curated set of structures suitable for fingerprinting has been produced after combining prior chemical knowledge into a list of validation criteria, implemented in the Privateer package [4]. The impact of chemical validation on the fingerprinting method and its practical applications will be discussed.

[1] R.P. Joosten et al, IUCrJ, 2014, 1:213-220.

[2] K. Cowtan, IUCrJ, Vol. 1, No. 6, 2014, p. 387-392.

[3] T. Lütteke, Acta Cryst., 2009, D65, 156-168.

[4] J. Agirre and K. Cowtan, Computational Crystallography Newsletter, Jan 2015, p. 10-12.

Keywords: carbohydrates, model building, conformation,
MS46. The use of resonant

scattering and diffraction for

the analysis of materials

Chairs: Jean-Louis Hodeau, Eugen Weschke

MS46-01 Application of resonant X-ray

scattering to thermoelectric materials that

contain elements with similar electron

counts

Oliver Oeckler ${ }^{1}$

1. Leipzig University, Faculty of Chemistry and Mineralogy, IMKM, Scharnhorststr. 20, 04275 Leipzig

email: oliver.oeckler@uni-leipzig.de

Layered compounds derived from $\mathrm{Sb}_{2} \mathrm{Te}_{3}$ and substituted variants thereof constitute one of the most important classes of efficient thermoelectric materials. They contain distorted rocksalt-type slabs separated by van der Waals gaps. In germanium antimony tellurides, for example, the thermoelectric properties can be enhanced by substituting Ge with Sn or Cd or by partially replacing $\mathrm{Sb}$ by $\mathrm{In}$. In addition, doping with $\mathrm{Ag}$ approaches other efficient materials like $\mathrm{AgSbTe}_{2}$. Precise structural data are essential for the understanding of structure-property relationships. In such compounds, elements with similar electron counts such as $\mathrm{Ag}, \mathrm{Cd}$, In, $\mathrm{Sn}, \mathrm{Sb}$ and possibly Te share the same Wyckoff positions which may additionally contain vacancies. Whereas vacancies tend to order, the other elements (often several of them) are usually mixed in different ratios on different positions. In some cases, periodic concentration gradients were observed.

In contrast to conventional structure analyses, resonant X-ray diffraction is an excellent tool to differentiate elements with usually low scattering contrast [1] and to reveal such partial ordering phenomena. Synchrotron data collected near the absorption edges are affected by dispersion correction terms $\Delta \mathrm{f}$ ' of up to 10 electrons. Joint refinements with multiple datasets collected at all $\mathrm{K}$ edges involved and at wavelengths far from edges are the most suitable strategy and avoid many shortcomings of "traditional" $\delta$ syntheses (which thus appear obsolete). A number of practical aspects were analyzed in detail [2] so that questions like "which is the optimal wavelength?" or "how is beamtime used most efficiently?" can unequivocally be answered. Various sources for $\Delta \mathrm{f}^{\prime}$ values were evaluated, the best being experimental values obtained from fluorescence data via the Kramers-Kronig transform and values that are refined using comparable "calibration compounds". The talk aims at giving practical guidelines rather than theoretical considerations and addresses both single-crystal and powder techniques. How can state-of-the-art precision be combined with "high throughput"? 
[1] J. L. Hodeau, V. Favre-Nicolin, S. Bos, H. Renevier, E. Lorenzo, J. F. Berar, Chem. Rev.. 2001, 101, 1843.

[2] S. Welzmiller, P. Urban, F. Fahrnbauer, L. Erra, O. Oeckler, J. Appl. Crystallogr. 2013, 46, 769.

Keywords: thermoelectrics, resonant scattering, element distribution

\section{MS46-O2 Electronic depth profiles with atomic layer resolution from Resonant X-ray Reflectivity}

Jochen Geck ${ }^{1,2}$, M. Zwiebler ${ }^{1}$, B. Gray ${ }^{3}$, J. Chakhalian ${ }^{3}$, J. Freeland $^{4}$, F. He ${ }^{5}$, A. Koitzsch ${ }^{1}$, P. Komissinskiy ${ }^{6}$, E. Schierle ${ }^{7}$, S. Sutarto $^{5}$, U. Treske ${ }^{1}$, M. Vafaee ${ }^{6}$, E. Weschke ${ }^{7}$, G. A. Sawatzky ${ }^{8}$, L. Alff ${ }^{6}$, S. Macke ${ }^{9,10}$, J. E. Hamann-Borrero ${ }^{1}$

1. Leibniz Institute for Solid State and Materials Research, IFW-Dresden, Helmholtzstr. 20, 01069 Dresden, Germany

2. Institut für Strukturphysik, Technische Universität Dresden, D-01062 Dresden, Germany

3. Department of Physics, University of Arkansas, Fayetteville, Arkansas 70701, USA

4. Advanced Photon Source, Argonne National Laboratory, Argonne, Illinois 60439, USA

5. Canadian Light Source, University of Saskatchewan, Saskatoon, Saskatchewan, S7N0X4, Canada

6. Institute of Materials Science, Technische Universität Darmstadt, 64287 Darmstadt, Germany

7. Helmholtz-Zentrum Berlin für Materialien und Energie, Albert-Einstein-Str. 15, D-12489 Berlin, Germany

8. Department of Physics and Astronomy, University of British Columbia, Vancouver, V6T1Z1, Canada

9. Max Planck-UBC Centre for Quantum Materials, Vancouver, V6T1Z1, Canada

10. Max Planck Institute for Solid State Research, Heisenbergstr. 1, 70569 Stuttgart, Germany

email: J.Geck@ifw-dresden.de

Resonant $\mathrm{X}$-ray reflectivity (RXR) provides a unique experimental tool to study the surface and buried interfaces of oxide heterostructures. The analysis of reflectivity data usually assumes homogeneous properties throughout the volume of the constituent materials, i.e., the internal atomic structure of these materials is usually neglected. However, when the x-ray energy is tuned to an absorption edge, this approximation can cease to provide a good description of the experiment, because lattice planes with and without the elements at resonance will interact with the photons very differently. As a result, RXR can also provide important and very useful information about a heterostructure at the atomic level. We have therefore developed a scheme for analyzing RXR-data, which takes the atomic structure of a material into account by "slicing" it into atomic planes with characteristic optical properties. Using $\mathrm{LaSrMnO}_{4}$ and $\mathrm{YBa}_{2} \mathrm{Cu}_{3} \mathrm{O}_{7}$ as examples, we discuss the implications of this approach. Our analysis not only allows to determine structural information such as interface terminations and stacking of atomic layers, but also enables to extract depth-resolved spectroscopic information with atomic resolution, thus further enhancing the capability of the technique to study emergent phenomena at surfaces and interfaces.

Keywords: oxide heterostructures, resonant soft x-ray scattering 